\title{
Ionophore-Based Biphasic Chemical Sensing in Droplet Microfluidics
}

\author{
Xuewei Wang,* Meng Sun, Stephen A. Ferguson, J. Damon Hoff, Yu Qin, Ryan C. Bailey, and \\ Mark E. Meyerhoff
}

\begin{abstract}
Droplet microfluidics is an enabling platform for high-throughput screens, single-cell studies, low-volume chemical diagnostics, and microscale material syntheses. Analytical methods for real-time and in situ detection of chemicals in the droplets will benefit these applications, but they remain limited. Reported herein is a novel heterogeneous chemical sensing strategy based on functionalization of the oil phase with rationally combined sensing reagents. Sub-nanoliter oil segments containing $\mathrm{pH}$-sensitive fluorophores, ionophores, and ion-exchangers enable highly selective and rapid fluorescence detection of physiologically important electrolytes $\left(\mathrm{K}^{+}, \mathrm{Na}^{+}\right.$, and $\mathrm{Cl}^{-}$) and polyions (protamine) in sub-nanoliter aqueous droplets. Electrolyte analysis in whole blood is demonstrated without suffering from optical interference from the sample matrix. Moreover, an oil phase doped with an aza-BODIPY dye allows indication of $\mathrm{H}_{2} \mathrm{O}_{2}$ in the aqueous droplets, exemplifying sensing of targets beyond ionic species.
\end{abstract}

Over the past two decades, droplet microfluidics has emerged as a unique subcategory of microfluidics that has found numerous applications in drug screening, directed evolution, single-cell analysis, and medical diagnosis, as well as for the synthesis of materials and molecules. ${ }^{[1]}$ In pressuredriven droplet microfluidics, an aqueous stream and a waterimmiscible oil stream intersect via a microchannel junction, such as a T-junction or a flow-focusing geometry. Discrete and monodisperse aqueous droplets or plugs are generated at femtoliter to nanoliter volumes at a frequency ranging from $\mathrm{Hz}$ to $\mathrm{kHz}$. This technique provides new opportunities to screen drug candidates and enzyme mutants in a reagentconservative and high-throughput fashion to perform chemical testing on small volumes of bodily fluids for minimally invasive diagnostics, and to study individual cells/bacteria/ viruses with low dilution factors and negligible liquid evaporation. The effective internal circulation within the flowing liquid segments and the favored interfacial mass transfer between microscale compartments also make droplet microfluidics an efficient platform for chemical synthesis and liquid-liquid extraction. ${ }^{[1 \mathrm{a}, 2]}$

[*] Dr. X. Wang, Dr. M. Sun, Dr. S. A. Ferguson, Dr. Y. Qin, Prof. R. C. Bailey, Prof. M. E. Meyerhoff Department of Chemistry, University of Michigan 930 N University, Ann Arbor, MI 48109 (USA) E-mail:wangxue@umich.edu

Dr. M. Sun, Dr. J. D. Hoff

Department of Biophysics, University of Michigan 930 N University, Ann Arbor, MI 48109 (USA) Supporting information and the ORCID identification number(s) for the author(s) of this article can be found under: https://doi.org/10.1002/anie.201902960.
In these applications, quantitative analysis of (bio)chemicals within droplets is essential for the determination of biomarker concentrations, the indication of enzymatic reactions/chemical interactions, examination of biochemical activities of cells/bacteria, and the monitoring of synthesis and extraction processes. Indeed, a wide variety of detection modalities including fluorescence spectroscopy, bright-field microscopy, infrared spectroscopy, surface-enhanced Raman spectroscopy, mass spectrometry, nuclear magnetic resonance spectroscopy, and electrochemistry have been employed in droplet microfluidics. ${ }^{[3]}$ Among them, fluorescence-based techniques are perhaps most common because their fast measurement rates can match the high droplet generation frequency and their high sensitivity is suited to the very low volumes of sample confined in the droplet. Current fluorescence-based tests are primarily restricted to enzyme assays (e.g., alkaline phosphatase ${ }^{[4]}$ and $\beta$-galactosidase ${ }^{[5]}$ ) using fluorogenic substrates, metabolite detection (e.g., L-lactate ${ }^{\left[{ }^{[6]}\right.}$ and ethanol ${ }^{[7]}$ ) using coupled enzyme reactions, and bioaffinity assays (e.g., immunoassay ${ }^{[8]}$ and DNA hybridization assays ${ }^{[9]}$ ) using fluorophore-labeled biomacromolecules. Also, these tests exclusively occur in the aqueous phase of the droplet microfluidics. Herein, we, for the first time, exploit the use of the oil segments in droplet microfluidics as a chemical sensing phase for targets in their adjacent aqueous droplets. This new strategy not only greatly extends the spectrum of analytes that can be quantified by the droplet microfluidics technology but also offers several key inherent advantages compared to chemical sensing in aqueous droplets. First, many host-guest recognition chemistries such as ionic complexation are favored in a low dielectric constant medium. ${ }^{[10]}$ Second, the low aqueous solubility is a challenge in the design of certain molecular probes, and is another reason that addition of an organic solvent into the assay solution is commonly practiced. In contrast, the design of highly hydrophobic probes is relatively easy by the introduction of hydrophobic moieties such as long alkyl chains. Third, the oil segments as sensors do not suffer from optical interference from the color and/or turbidity of the aqueous sample matrix. Lastly, fabrication and operation of the microfluidic chip are simplified because there is no need to use an additional aqueous stream to introduce sensing reagents into the sample phase.

The first targets of our methodology are electrolytes, such as $\mathrm{K}^{+}, \mathrm{Na}^{+}$, and $\mathrm{Cl}^{-}$. Quantification of these ions is important for medical diagnostics, monitoring of ion-channel functions, and the study of ion binding to biomacromolecules. Droplet microfluidics may greatly benefit these applications, but in situ sensing of these electrolytes has not been reported in droplet microfluidics. The most successful technique for electrolyte analysis is the use of ionophore-based ion- 
selective sensors (electrodes and optodes). ${ }^{[11,12]}$ Interestingly, these sensors rely on a water-immiscible organic phase such as plasticized membranes or micro/nanoparticles to test analytes in an aqueous sample, which resembles the biphasic scaffold of droplet microfluidics. Therefore, we take advantage of this similarity to incorporate ion-sensing functionalities into droplet microfluidics. Notably, there is one report on the use of an ion-selective electrode to detect ions $\left(\mathrm{Mg}^{2+}\right)$ in a continuous aqueous phase separated from the segmented flow, ${ }^{[13]}$ but the requirement of phase separation scarifies most attractive features, such as the throughput and the capability of droplet microfluidics to study individual cells.

Scheme 1 shows the principle of optical sensing of cations $\left(\mathrm{K}^{+}\right.$as an example) in droplet microfluidics. Highly hydrophobic sensing chemicals including a $\mathrm{pH}$ indicator dye
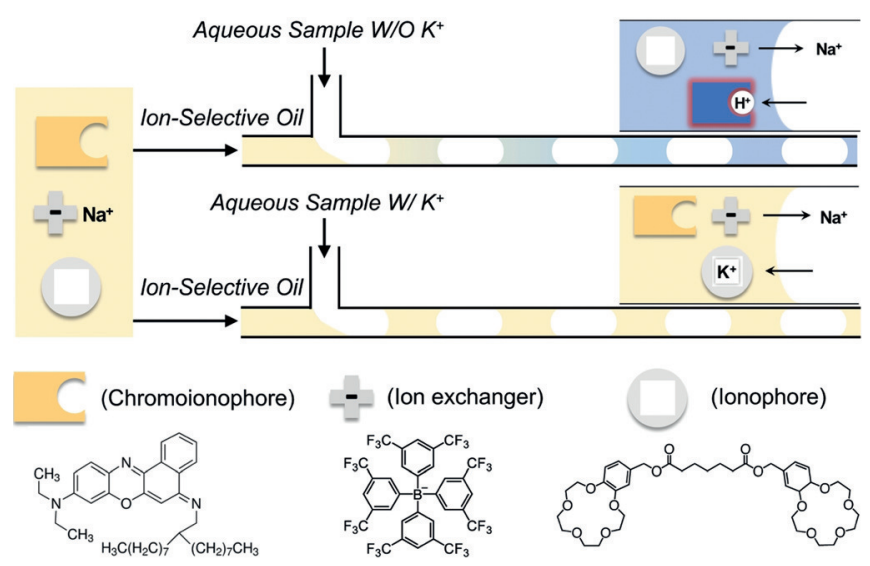

Scheme 1. Operation principle of biphasic $\mathrm{K}^{+}$sensing platform in droplet microfluidics.

(fluorescent chromoionophore) as an optical read-out element, an ionophore as the ion recognition element, and a cation exchanger to prevent interference from anions are dissolved into a water-immiscible oil. By using two infusion pumps, this oil phase is merged with an aqueous solution within a T-junction microchannel under conditions that generate segmented flow. For a buffered aqueous sample without the target cations, protons from this sample can transfer into the oil phase and protonate a chromoionophore having an appropriate $\mathrm{p} K_{\mathrm{a}}$. This process is accompanied by expulsion of the hydrophilic $\mathrm{Na}^{+}$from the oil segments into the aqueous droplets to maintain electroneutrality of the oil. In contrast, when the aqueous sample contains target cations, these ions will be extracted into the oil segments because of the very high binding affinity of the ionophore to the target ion. This ionic complexation process competes with the protonation of the chromoionophore, and the degree of protonation determines the absorbance and fluorescence properties of the $\mathrm{pH}$-sensitive dye in the oil segments.

The color and fluorescence of the oil phase can be monitored by high-speed bright-field video and laser-induced fluorescence, respectively, at the distal end of the downstream channel, which is $3.5 \mathrm{~cm}$ from the junction. As can be seen from Figure $1 \mathrm{~A}$, the oil segments are blue when the $\mathrm{K}^{+}$
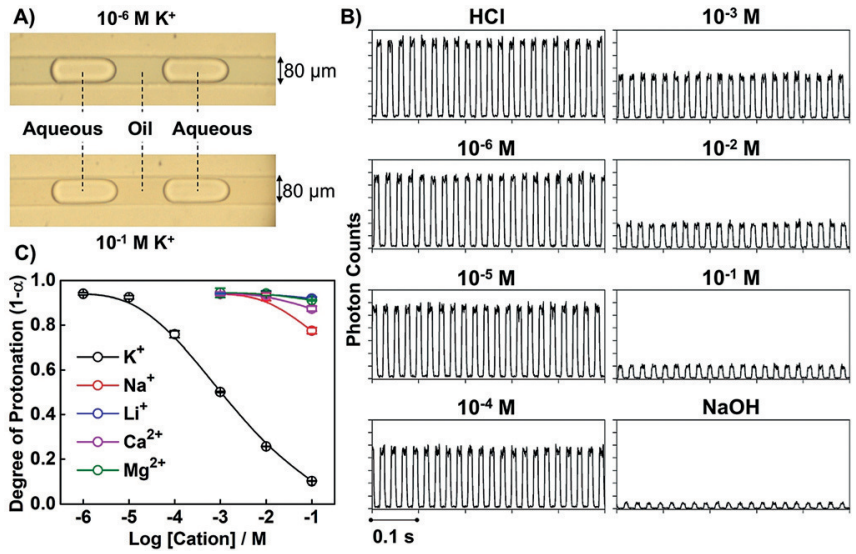

Figure 1. A) Bright-field images of the segmented fluids in the presence of $10^{-6} \mathrm{M}$ and $10^{-1} \mathrm{M} \mathrm{KCl}$ in the aqueous phase. The used oil is dioctyl sebacate, and the chip is made of PDMS. B) Fluorescence trace of the segmented flow in the presence of different concentrations of $\mathrm{KCl}$ in Tris- $\mathrm{HCl}$ buffer at $\mathrm{pH} 7.4$ and $0.1 \mathrm{M} \mathrm{HCl}$ or $\mathrm{NaOH}$. C) Response curve of this sensing method toward different cations based on the 0.5 -s test (data points are average \pm SD for $n=3$ measurements).

concentration is low and become orange for a higher $\mathrm{K}^{+}$ concentration, which results from the protonated and deprotonated chromoionophore, respectively (see Figure S1 A for the absorbance spectra). The color difference is small because of the short optical path length $(40 \mu \mathrm{m})$ in the microchannel. Therefore, we employ fluorescence spectroscopy to perform quantitative ion analysis. Based on the fluorescence spectra of the protonated and deprotonated chromoionophore in bulk dioctyl sebacate (see Figure S1 B in the Supporting Information), an excitation laser at $630 \mathrm{~nm}$ and an emission wavelength band of $673-738 \mathrm{~nm}$ were chosen. As shown in Figure 1B, significant fluorescence is observed only from the oil segments phase because of the high hydrophobicity of the dye (chromoionophore III, $\log P=10.5$, from ChemDraw). A higher concentration of $\mathrm{K}^{+}$in the aqueous phase leads to a lower fraction of protonated chromoionophore molecules and yields less fluorescence in the oil segment because of the unfavored charge transfer between the donor and acceptor groups in the chromoionophore. The mean fluorescence signal $(F)$ of all oil segments during a 0.5 second period is obtained from a Gaussian fit of the photon intensity (photons per second) emitted from the oil phase. The fluorescence of the completely protonated $\left(F_{\max }\right)$ and deprotonated $\left(F_{\min }\right)$ chromoionophore in the segmented oil is acquired when the aqueous phase consists of $0.1 \mathrm{M} \mathrm{HCl}$ and $0.1 \mathrm{M} \mathrm{NaOH}$, respectively. The degree of protonation $(1-\alpha)$ is then calculated by the following equation:

$1-\alpha=\frac{\left[\mathrm{CH}^{+}\right]}{\left[\mathrm{C}_{\mathrm{T}}\right]}=1-\left(\frac{F_{\max }-F}{F_{\max }-F_{\min }}\right)$

where $\left[\mathrm{CH}^{+}\right]$is the concentration of the protonated chromoionophore, and $\left[\mathrm{C}_{\mathrm{T}}\right]$ is the total concentration of the chromoionophore. As is convention in the field of ionselective optodes, ${ }^{[12]}$ we plot the degree of protonation against the concentration of the analyte ion in the aqueous phase to 
create the calibration curve (Figure $1 \mathrm{C}$ ). Like traditional ionselective optodes, ${ }^{[12]}$ this sensing method has a wide dynamic range covering several orders of magnitude of analyte concentration. Half protonation of the chromoionophore occurs at around $10^{-3} \mathrm{M} \mathrm{K}^{+}\left(\left[\mathrm{K}^{+}\right] /\left[\mathrm{H}^{+}\right]=10^{4.4}\right)$, which represents a sensitivity similar to those of other $\mathrm{K}^{+}$optodes. ${ }^{[14]}$ The standard deviation shown in Figure $1 \mathrm{C}$ could be translated into a concentration error of $\pm 3 \%$ for $10^{-3} \mathrm{M} \mathrm{K}^{+}$and $\pm 1 \%$ for $10^{-2} \mathrm{M} \mathrm{K}^{+}$based on the linear regression formula shown in Figure S2. Furthermore, because of the specificity of the ionophore, this system is at least 1000 -fold more sensitive toward $\mathrm{K}^{+}$than other cations including $\mathrm{Na}^{+}, \mathrm{Li}^{+}, \mathrm{Ca}^{2+}$, and $\mathrm{Mg}^{2+}$.

One difference of this sensing scheme compared to traditional ion-selective optodes is the equal volume of the sample phase and the sensing phase. Depletion of the analyte in the sample becomes possible, and is similar to the ionselective nanooptodes operating in exhaustive sensing mode. ${ }^{[15]}$ According to the deprotonation of the chromoionophore, $4.5 \%$ and $0.7 \%$ of $\mathrm{K}^{+}$is extracted into the oil phase when the sample has $10^{-3} \mathrm{M}$ and $10^{-2} \mathrm{M}$ of $\mathrm{KCl}$, respectively.

Based on the flow rate $\left(2 \mu \mathrm{L} \mathrm{min}^{-1}\right.$ for each phase $)$ and the frequency of segment generation (ca. 41 segments per second for each phase), the size of each oil segment and each aqueous droplet is estimated to be about $0.8 \mathrm{~nL}$. A test time of $0.5 \mathrm{~s}$ corresponds to an aqueous sample volume of about $17 \mathrm{~nL}$ and 21 measurement events. Taking the $10^{-3} \mathrm{M} \mathrm{KCl}$ sample as an example, we obtained a relative standard deviation of only $1.2 \%$ for the averaged fluorescence from the 21 oil segments. The negligible variation between different oil segments and their fluorescence intensities may allow further reduction of the sample volume and the measurement time. The use of an ultrasmall volume of sample to perform a test is critical for the concept of minimally invasive diagnostics such as those based on fingerstick blood sampling or a microneedle-based painless blood draw, especially when a large number of parameters are to be quantitated from a single sample.

Figure 2 shows the fluorescence change as a function of distance along the channel after droplet generation. When the aqueous phase has $10^{-6} \mathrm{M} \mathrm{KCl}$, the fluorescence of the oil phase reaches $95 \%$ equilibrium after $2.5 \mathrm{~cm}$ of travel, corresponding to the process of chromoionophore protonation. This $2.5 \mathrm{~cm}$ distance takes about 1.8 seconds to traverse based on a moving velocity of about $170 \mu \mathrm{m}$ per $12 \mathrm{~ms}$ (each segment). For a high concentration of $\mathrm{K}^{+}$, the chromoionophore in the oil phase only needs to be partially protonated or remains deprotonated, and takes a shorter time to reach equilibrium. Such response times are more than 50-fold shorter than conventional polymeric membrane-type ionselective optodes with film thicknesses of a couple of micrometers $^{[14 a]}$ and microsphere-type ion-selective optodes with diameters of about $20 \mu \mathrm{m} .{ }^{[14 \mathrm{~b}]}$ This method is also faster than ion sensing in parallel flow-based microfluidics. ${ }^{[16]}$ The very fast response time is likely related to the lower viscosity of the pure liquid oil phase compared to the commonly used polymer-plasticizer mixture, and the enhanced convection within both aqueous droplets and oil segments resulting from the friction-induced internal flow circulation. ${ }^{[17]}$
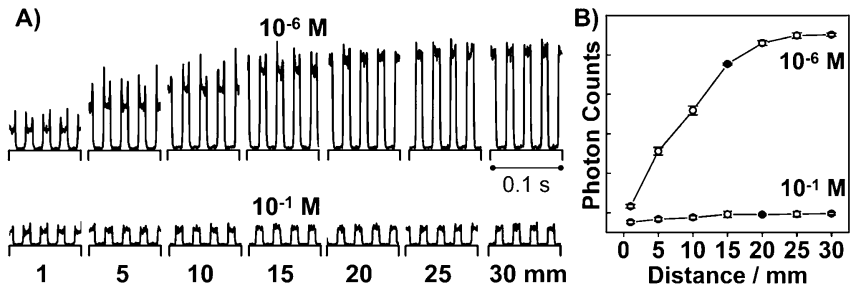

Figure 2. A) Fluorescence trace of the segmented flow at different locations along the channel after the merging junction. B) Mean photon intensity of the oil segments as a function of distance from the junction (data points are average \pm SD for $n=3$ measurements).

Traditional fluorescent probes are usually susceptible to optical interference from colored and/or turbid samples such as whole blood. Indeed, although the formation of stable blood droplets has been reported in an image-based study of blood coagulation, ${ }^{[18]}$ optical chemical analysis in whole blood has not, to the best of our knowledge, been reported previously in droplet microfluidics. Interrogation of such complicated samples is also quite challenging for other analytical techniques such as mass spectrometry and Raman spectroscopy. In contrast, our biphasic sensing scheme uses physically separated sensors and samples. Since both the laser illumination and the fluorescence collection are perpendicular to the PDMS chip, the signal generated from the oil segment can be monitored without suffering from optical interference from the blood. As shown in Figure 3, the blood

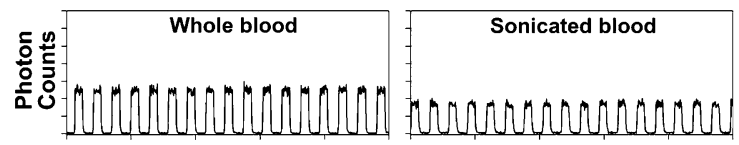

Figure 3. Fluorescence trace of the segmented flow for analysis of whole blood and sonicated whole blood. A 1:1 dilution of the whole blood with $0.1 \mathrm{M}$ Tris- $\mathrm{HCl}$ buffer at $\mathrm{pH} 7.4$ was employed to control the sample $\mathrm{pH}$.

droplets do not exhibit any fluorescence under the employed experimental conditions, but they are able to induce fluorescence in the oil segments based on ion extraction. The $\mathrm{K}^{+}$ concentration in the blood sample is calculated to be $2.6 \pm$ $0.4 \mathrm{~mm}$, which reasonably matches the concentration of $2.2 \mathrm{~mm}$ obtained by a commercial blood gas/electrolyte analyzer (see Figure S2). The error may be related to spontaneous hemolysis of blood in the microchannel. When the blood sample was sonicated for 5 seconds before being introduced into the microfluidics chip, significantly reduced fluorescence is observed from the oil segments due to the release of $\mathrm{K}^{+}$from the broken blood cells (Figure 3 ). Extraction of lipophilic compounds from biological samples into the oil segment might be another source of error. Such non-selective extraction could be prevented by using a fluorous oil phase with perfluorinated sensing chemicals.

One feature of ionophore-based ion-selective detection using combined reagents is that the selectivity and the sensitivity can be adjusted by using different sensing reagents and different ratios of those reagents. For example, the use of 
a $\mathrm{Na}^{+}$ionophore and a tetraphenylborate-type cation exchanger in its potassium salt form renders the oil segments selective toward $\mathrm{Na}^{+}$(see Figure S3). By using a mercuracarborand "anti-crown ether" ionophore for $\mathrm{Cl}^{-}$, a quaternaryammonium-type anion exchanger, and a less basic chromoionophore (chromoionophore I), fluorescent sensing of $\mathrm{Cl}^{-}$can also be achieved (see Figure S4). In addition, highly selective ionophores for other inorganic cations such as $\mathrm{Ca}^{2+}, \mathrm{Mg}^{2+}$, $\mathrm{Pb}^{2+}, \mathrm{Zn}^{2+}, \mathrm{Cd}^{2+}, \mathrm{Hg}^{2+}, \mathrm{Ag}^{+}$, and other inorganic anions such as $\mathrm{F}^{-}, \mathrm{NO}_{3}^{-}, \mathrm{NO}_{2}^{-}, \mathrm{SO}_{4}{ }^{2-}, \mathrm{CO}_{3}{ }^{2-}$ are available. ${ }^{[11]}$ A wide range of organic ions (e.g., creatinine and choline derivatives) ${ }^{[19]}$ polyions (e.g., protamine, heparin, and DNA) ${ }^{\left[{ }^{[0]} \text { and }\right.}$ even uncharged organic molecules (e.g., phenols, boronic acids, saccharides, and $\left.\mathrm{H}_{2} \mathrm{O}_{2}\right)^{[21]}$ have also been detected by biphasic sensors. Therefore, the oil-based sensing concept promises to bring a wealth of new analytical capabilities to the droplet microfluidics and advance its applications.

Herein, one additional example of this new chemical sensing approach is highlighted by detection of protamine, an arginine-rich protein with 21 positive charges. The oil phase is 1,2-dichloroethane with chromoionophore I and dinonylnaphthalene sulfonic acid (DNNSH). Selective extraction of protamine over singly charged cations into the oil segments is driven by the strong cooperative ion-pairing interaction between one multiply charged protamine and multiple singly charged DNNS anions. ${ }^{[20]}$ Such extraction competes with protonation of the chromoionophore and induces decreased fluorescence in the oil segments. As shown in Figure 4, this method exhibits a fluorescent response toward

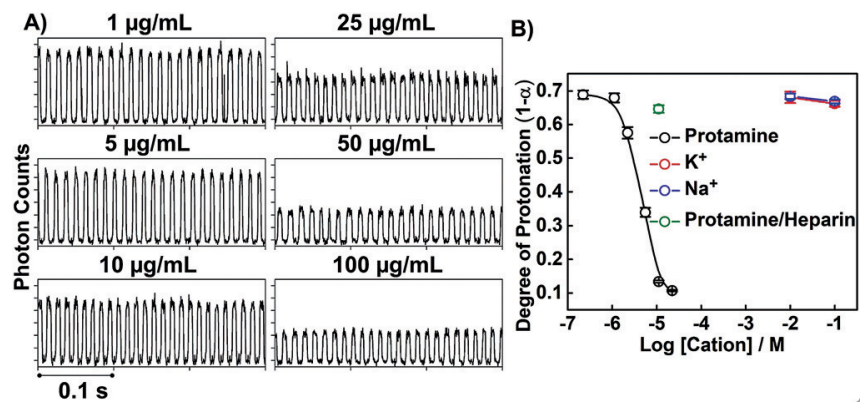

Figure 4. A) Fluorescence trace of the segmented flow in the presence of different concentrations of protamine in $50 \mathrm{~mm}$ Tris- $\mathrm{HCl}$ buffer at $\mathrm{pH} 7.4$ as the aqueous phase (excitation: $630 \mathrm{~nm}$; emission: $673-$ $738 \mathrm{~nm}$ ). B) Calibration curves for protamine and small cations. The green circle is the response toward a $50 \mu \mathrm{g} \mathrm{mL}^{-1}$ protamine solution containing $12 \mathrm{U} \mathrm{mL}^{-1}$ heparin (data points are average $\pm \mathrm{SD}$ for $n=3$ measurements).

protamine over a dynamic range of 1 to $100 \mu \mathrm{g} \mathrm{mL}^{-1}$, which is comparable to other protamine-sensitive optodes, but is achieved here on a much shorter timescale (ca. $1.2 \mathrm{~s}$ vs. $>10 \mathrm{~min}) .^{[20 \mathrm{~b}, \mathrm{c}]}$ The strong ion association interaction also makes conventional polyion-selective sensors irreversible. ${ }^{[20]}$ However, in our sensing scheme, every oil segment is only used for one measurement, and fresh oil segments are generated continuously. Therefore, there is no requirement for reversibility of the biphasic sensing chemistry, which is another distinct advantage of this droplet microfluidics-based sensing scheme. If the protamine is neutralized by polyanions such as heparin, the fluorescence response is prevented because the complexed polycations are no longer able to form ion pairs with DNNS anions (Figure 4B, green circle). Based on this mechanism, we are currently working on continuous monitoring of blood heparin levels using droplet microfluidics, which would be useful during extracorporeal procedures (e.g., cardiopulmonary bypass surgery) to determine the actual concentration of this anticoagulant in blood. Moreover, this same polyion-sensing method has other indirect applications such as enzyme assays, enzyme inhibitor/activator tests, and aptasensing ${ }^{[20]}$ which might now be integrated into a droplet microfluidic platform.

In another proof-of-principle example, the chemical sensing of neutral species is demonstrated using droplet microfluidics. The limited aqueous solubility is an obstacle in the application of some BODIPY and azaBODIPY dyes. However, we successfully use a boronic acid functionalized azaBODIPY dye in the oil phase to detect $\mathrm{H}_{2} \mathrm{O}_{2}$, an important reactive oxygen species, in the aqueous droplet based on the oxidative conversion of boronic acids into phenols in the oil phase (see Figure S5). ${ }^{[21 d]}$

In summary, the oil phase in droplet microfluidics provides a promising scaffold for sensing of ionic, polyionic, and non-ionic species in aqueous sample droplets. This sensing scheme is fast, reagent-economic, and compatible with complicated sample matrices. The ultimate goal of this endeavor lies in the clinical analysis of chemical species using ultrasmall volumes of bodily fluids, as well as the highthroughput screening of drugs that target ion channels on individual whole cells, both of which are not readily achievable by current analytical techniques.

\section{Acknowledgements}

This work was financially supported by the Exercise and Sport Science Initiative Pilot Grant at the University of Michigan (ESSI-2018-6) and National Institutes of Health (CA191186). We also thank the SMART Center of the University of Michigan, seeded by NSF MRI-ID award DBI-0959823.

\section{Conflict of interest}

The authors declare no conflict of interest.

Keywords: analytical methods - droplet microfluidics . ionophores $\cdot$ molecular recognition $\cdot$ sensors

How to cite: Angew. Chem. Int. Ed. 2019, 58, 8092-8096 Angew. Chem. 2019, 131, 8176-8180

[1] a) S. Y. Teh, R. Lin, L. H. Hung, A. P. Lee, Lab Chip 2008, 8, $198-220$; b) M. T. Guo, A. Rotem, J. A. Heyman, D. A. Weitz, Lab Chip 2012, 12, 2146-2155; c) L. R. Shang, Y. Cheng, Y. J. Zhao, Chem. Rev. 2017, 117, $7964-8040$.

[2] a) P. Mary, V. Studer, P. Tabeling, Anal. Chem. 2008, 80, $2680-$ 2687; b) S. Mashaghi, A. M. Van Oijen, Sci. Rep. 2015, 5, 11837. 
[3] a) Y. Zhu, Q. Fang, Anal. Chim. Acta 2013, 787, 24-35; b) E. Y Basova, F. Foret, Analyst 2015, 140, $22-38$; c) A. Kalantarifard, A. Saateh, C. Elbuken, Chemosensors 2018, 6, 23.

[4] A. Huebner, L. F. Olguin, D. Bratton, G. Whyte, W. T. S. Huck, A. J. De Mello, J. B. Edel, C. Abell, F. Hollfelder, Anal. Chem. 2008, $80,3890-3896$.

[5] S. L. Sjostrom, H. N. Joensson, H. A. Svahn, Lab Chip 2013, 13 , $1754-1761$.

[6] B. L. Wang, A. Ghaderi, H. Zhou, J. Agresti, D. A. Weitz, G. R Fink, G. Stephanopoulos, Nat. Biotechnol. 2014, 32, 473-478.

[7] S. Abalde-Cela, A. Gould, X. Liu, E. Kazamia, A. G. Smith, C. Abell, J. R. Soc. Interface 2015, 12, 20150216.

[8] N. Shembekar, H. Hu, D. Eustace, C. A. Merten, Cell Rep. 2018 $22,2206-2215$.

[9] A. T. H. Hsieh, P. J. H. Pan, A. P. Lee, Microfluid. Nanofluid. 2009, 6, $391-401$

[10] a) J. W. Jones, H. W. Gibson, J. Am. Chem. Soc. 2003, 125, 7001 7004; b) M. J. Langton, C. J. Serpell, P. D. Beer, Angew. Chem. Int. Ed. 2016, 55, 1974-1987; Angew. Chem. 2016, 128, 2012 2026.

[11] P. Buhlmann, L. D. Chen in Supramolecular Chemistry: From Molecules to Nanomaterials (Eds.: J. W. Steed, P. Gale), Wiley, New York, 2012, pp. 2539-2579.

[12] a) E. Bakker, P. Bulhmann, E. Pretsch, Chem. Rev. 1997, 97, 3083-3132; b) X. Xie, E. Bakker, Anal. Bioanal. Chem. 2015 407, 3899-3910.

[13] Z. Han, Y. Y. Chang, S. W. Au, B. Zheng, Chem. Commun. 2012 , 48, $1601-1603$.

[14] a) M. Bamsey, A. Berinstain, M. Dixon, Anal. Chim. Acta 2012, 737, $72-82$; b) N. Ye, K. Wygladacz, E. Bakker, Anal. Chim. Acta 2007, 596, 195-200.
[15] X. Xie, J. Zhai, G. A. Crespo, E. Bakker, Anal. Chem. 2014, 86, $8770-8775$

[16] H. Hisamoto, T. Horiuchi, M. Tokeshi, A. Hibara, T. Kitamori, Anal. Chem. 2001, 73, 1382-1386.

[17] H. Song, D. L. Chen, R. F. Ismagilov, Angew. Chem. Int. Ed. 2006, 45, 7336-7356; Angew. Chem. 2006, 118, 7494-7516.

[18] H. Song, H. W. Li, M. S. Munson, T. G. Van Ha, R. F. Ismagilov, Anal. Chem. 2006, 78, 4839-4849.

[19] a) T. Guinovart, D. Hernández-Alonso, L. Adriaenssens, P. Blondeau, M. Martínez-Belmonte, F. X. Rius, F. J. Andrade, P. Ballester, Angew. Chem. Int. Ed. 2016, 55, 2435-2440; Angew. Chem. 2016, 128, 2481-2486; b) J. Ampurdanés, G. A. Crespo, A. Maroto, M. A. Sarmentero, P. Ballester, F. X. Rius, Biosens. Bioelectron. 2009, 25, 344-349.

[20] a) M. E. Meyerhoff, B. Fu, E. Bakker, J. H. Yun, V. C. Yang, Anal. Chem. 1996, 68, 168A-175A; b) S. B. Kim, T. Y. Kang, H. C. Cho, M. H. Choi, G. S. Cha, H. Nam, Anal. Chim. Acta 2001, 439, 47-53; c) X. Wang, M. Mahoney, M. E. Meyerhoff, Anal. Chem. 2017, 89, 12334-12341; d) S. A. Ferguson, M. E. Meyerhoff, Sens. Actuators B 2018, 272, 643-654.

[21] a) X. Wang, Z. Ding, Q. Ren, W. Qin, Anal. Chem. 2013, 85, 1945 -1950; b) X. Wang, D. Yue, E. Lv, L. Wu, W. Qin, Anal. Chem. 2014, 86, 1927-1931; c) J. Zhai, T. Pan, J. Zhu, Y. Xu, J. Chen, Y. Xie, Y. Qin, Anal. Chem. 2012, 84, 10214-10220; d) Y. Liu, J. Zhu, Y. Xu, Y. Qin, D. Jiang, ACS Appl. Mater. Interfaces $\mathbf{2 0 1 5}, 7,11141-11145$.

Manuscript received: March 8, 2019

Revised manuscript received: April 14, 2019

Accepted manuscript online: April 17, 2019

Version of record online: May 8, 2019 